each channel. Because of the central role played by the semiclassical approximation in heavy ion physics, this error has serious consequences, both conceptual as well as practical (for example, the incorrect referencing in p265 to standard computer codes).

On p398 the absolute value of the cross section associated with twonucleon transfer reactions is discussed. The author should have noted that the cross section increases, due to the inclusion of continuum states in the calculation of the corresponding form factors, not by a factor of 25 but rather by a factor of 2 . It is correct that in the reference quoted the value of 25 appears. However, in a later publication the same authors acknowledge an error in their computer code, and the new revised value is given. By the way,

\section{Digital image processing}

Digital Image Processing and Analysis. Edited by J. C. Simon and A. Rosenfeld. Pp. 513. (Sithoff and Noordhoff: Leiden, The Netherlands, 1977.) \$39.50. Digital Image Processing. By W. K. Pratt. Pp. 750. (Wiley: New York and Chichester, UK, 1978.) $£ 20.80$.

IN 1976, a NATO Advanced Study Institute was held at Bonas, in south-western France. Digital Image Processing and Analysis contains 38 papers delivered at that meeting, printed from authors' typescripts. Conference proceedings are now commonly produced three or four months after submission of the manuscripts and there really is no excuse for allowing nearly two years to elapse, especially in such a fast-moving field as image processing. There is thus not much in this book that has not already appeared in print elsewhere.

The papers are a mixture of introductory surveys by well known scientists and shorter contributions. Of the former, there is little that is not better set out elsewhere, in textbooks such as Pratt's, for example, or Rosenfeld's own volume (for review, see Nature 264, 142; 1976); of the other papers, a few contain original material-those on recursive filtering in particular-but most of the material duplicates papers published more rapidly and accessibly in journals.

One redeeming feature of the book is the breadth of coverage. Image enhancement and mensuration are covered as well as pattern recognition, and it is certainly healthy to have these intimately related topics in the same volume. Nevertheless budget-conscious librarians can reasonably regard this volume-unlike several other volumes in the NATO ASI seriesas dispensable.

Professor Pratt's volume on Digital this value of about 2 is similar to the value found by Feng et al., the reference for which is quoted in p422 of this book, also in connection with the absolute cross section of two-particle transfer reactions. It is probably worth noting that an enhancement of the order of 2-4 would be expected from the experience accumulated on this subject in two-particle transfer reactions induced by light projectiles.

According to the cover jacket the book is aimed at graduate students. Let us hope that the excitement which awaits these graduate $s$ udents entering the field of heavy ion physics, will not be diminished by the rather confusing picture given by this book.

Ricardo A. Broglia is at the Niels Bohr Institute, University of Copenhagen, Denmark.

Image Processing is a long and detailed textbook, which has grown out of his image processing course given to electrical engineering students in the University of Southern California. The result of several years' work, it is clear, detailed and authoritative, and-a very valuable feature--the author is aware which topics are difficult to grasp and which can be treated in a reasonably summary fashion. Furthermore, it contains sections on colourimetry and the visual aspects of image appreciation as well as the formal mathematics of image processing. There are sections on image coding and on comparatively recent

\section{Magnetospheric physics}

Geomagnetic Diagnosis of the Magnetosphere. Physics and Chemistry in Space, Vol. 9. By A. Nishida. Pp.256. (Springer: New York, Heidelberg and Berlin, 1978.)

THE title of this book emphasises the use of geomagnetic data from instruments at ground level for sensing dynamic phenomena occurring far out in the magnetosphere. The value of such observations is also stressed in the preface, and the preface explains that the pursuit of this theme has evolved into an "introduction to magnetospheric physics". In both respects the book is successful, demonstrating how magnetospheric physics can be split with only tolerable damage at a timescale of about one second. Although processes typified by anomalous diffusion are important for the slow disturbances, it is not necessary to discuss the mechanisms, which involve higher frequencies.

The crudest approximation in plasma physics is hydromagnetics and this provides a useful start. Page 1 is indeed a sudden commencement, but coher- additions to the field of image processing, such as texture analysis.

The book has the great merit of not only describing the various techniques that are in use but also explaining their purpose and their role in the overall development of this complex subject. As we should expect from a member of the Image Processing Institute, the algebraic aspects of image processing theory are explained clearly and fully but they are not allowed an excessive amount of space.

One criticism can be made, and it is common to most of the recent texts on image processing: the field of electron microscopy, in which the importance of digital image processing has been increasingly recognised during the past few years, is scarcely ever mentioned. This is regrettable, for not only have electron micrographs benefited from some of the techniques described here - originally intended for other types of image--but some quite new procedures have been developed which may well be of interest to students of image processing in general.

This is an extremely well organised and lucid textbook. Despite the author's modest claim that is intended for use as a course text, many workers already familiar with much of the material will find it useful.

P. W. Hawkes

P. W. Hawkes is Maître de Recherches in the CNRS Laboratory of Electron Optics, Toulouse, France.

ence persists through to the last pulsating chapter, another hydromagnetic success. Much of the intervening chapters is related to reconnection and to particle motion. Dr Nishida has digested a mass of literature on observations and has produced a clear survey, which provides relief after the disjointedness of conference reports. Magnetosphere-ionosphere interactions are included and these sections are of particular relevance to observations using the ground-based incoherent scatter technique; this is timely as a large installation, EISCAT, is now being. built.

As an introduction to magnetospheric physics this monograph fills a need; it is splendid for new graduate students or others entering the field. It shows the current rapid progress, being made but warnings such as "the problem of the modelling of the dayside reconnection should not be considered to have been settled" are too weak, and more emphasis might have been given to existing controversies. For the substantial research still needed this book will provide a baseline.

\section{J. W. Dungey}

7. W. Dungey is a Professor of Physics at Imperial College, University of London, $U K$. 